\title{
Mycoplasma pneumoniae-associated Mucositis: A Recently Described Entity
}

\author{
Inês Zão ${ }^{1}$, Fani Ribeiro ${ }^{1}$, Valter Rocha ${ }^{2}$, Pedro Neto ${ }^{1}$, Carla Matias ${ }^{1}$, Gorete Jesus ${ }^{1}$ \\ ${ }^{1}$ Internal Medicine Department, Centro Hospitalar do Baixo Vouga, Aveiro, Portugal \\ ${ }^{2}$ Intensive Care Department, Centro Hospitalar Entre Douro e Vouga, Santa Maria da Feira, Portugal
}

\section{Received: $15 / 10 / 2018$}

Accepted: 09/11/2018

Published: 20/11/2018

\begin{abstract}
How to cite this article: Zão I, Ribeiro F, Rocha V, Neto P, Matias C, Jesus G. Mycoplasma pneumoniae-associated mucositis: a recently described entity.
\end{abstract} EJCRIM 2018;5: doi:10.12890/2018_000977.

Conflicts of Interests: The Authors declare that there are no competing interests.

This article is licensed under a Commons Attribution Non-Commercial 4.0 License

\section{ABSTRACT}

Mycoplasma pneumoniae (MP) is a common cause of respiratory infections and can be associated with extrapulmonary complications. MP mucositis has recently been described as a distinct endemic clinical entity called Mycoplasma pneumoniae-induced rash and mucositis (MIRM). The authors present the case of a 46-year-old man with atypical pneumonia associated with exuberant mucositis, conjunctival hyperaemia and positive serological assays for MP IgM. The patient was treated with azithromycin and systemic corticosteroid therapy. Supportive care including pain management, intravenous hydration and mucosal care was also given. There was complete resolution of the pneumonia and mucositis. The presence of atypical pneumonia with mucosal involvement without cutaneous lesions and a favourable clinical evolution led to the diagnosis of MIRM.

\section{LEARNING POINTS}

- Mycoplasma pneumoniae infection can be associated with mucocutaneous lesions. A new entity called Mycoplasma pneumoniae-induced rash and mucositis (MIRM) has been recently described. The mucocutaneous involvement associated with MIRM is predominantly mucositis with scarce or absent cutaneous expression.

- The clinical presentation, pathophysiology and disease outcomes of MIRM distinguish it from Stevens-Johnson syndrome/toxic epidermal necrolysis and erythema multiform.

- MIRM has an overall favourable prognosis as the majority of patients recover without sequalae and recurrence is rare.

\section{KEYWORDS}

Mycoplasma pneumoniae, mucositis, conjunctival hyperaemia, atypical pneumonia

\section{CASE DESCRIPTION}

A 46-year-old man who worked in forestry was admitted to the emergency department (ED) with a 12-day history of fever, malaise, headache, rhinorrhoea, mildly productive cough and odynophagia. He also reported a 2- day history of bilateral conjunctival hyperaemia and exuberant mucositis associated with a decrease in food intake due to odynophagia. Ten days before admission the patient had been prescribed amoxicillin/clavulanic acid by his general practitioner. On admission to the ED, the patient was haemodynamically stable, eupnoeic and febrile (tympanic temperature of $38.5^{\circ} \mathrm{C}$ ), with exuberant mucositis and bilateral conjunctival hyperaemia without cutaneous lesions or lesions in the genital region. Pulmonary auscultation presented bibasilar crackles. 


\section{METHODS AND PROCEDURES}

Blood examinations showed an elevated C-reactive protein (CRP) and erythrocyte sedimentation rate (ESR) (CRP $15.32 \mathrm{mg} / \mathrm{dl}$, ESR $65 \mathrm{~mm}$ ),

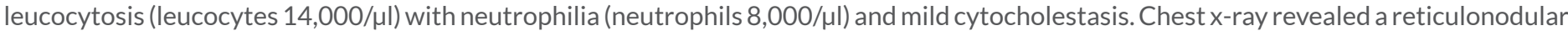
infiltrate in both pulmonary bases which corresponds to an atypical pneumonia pattern. The remaining examinations showed negative immunological screening, a non-reactive VDRL (Venereal Disease Research Laboratory) test, and negative screening tests for hepatitis B and $\mathrm{C}$ viruses, human immunodeficiency virus, cytomegalovirus, Epstein-Barr virus, Chlamydophila pneumoniae, Rickettsia conorii, Coxiella burnetii, Borrelia burgdorferi, Leptospira and Legionella pneumophila. Serology-based assays were positive for Mycoplasma pneumoniae (MP) IgM. The otorhinolaryngological examination revealed extensive mucositis with confluent ulcers on the oropharynx and hypopharynx (grade 3 mucositis) (Fig. 1). The ophthalmological examination revealed exuberant conjunctival hyperaemia, follicles on the tarsal conjunctiva, inflammatory membranes adherent to the conjunctiva and superficial punctate keratitis (Fig. 2).

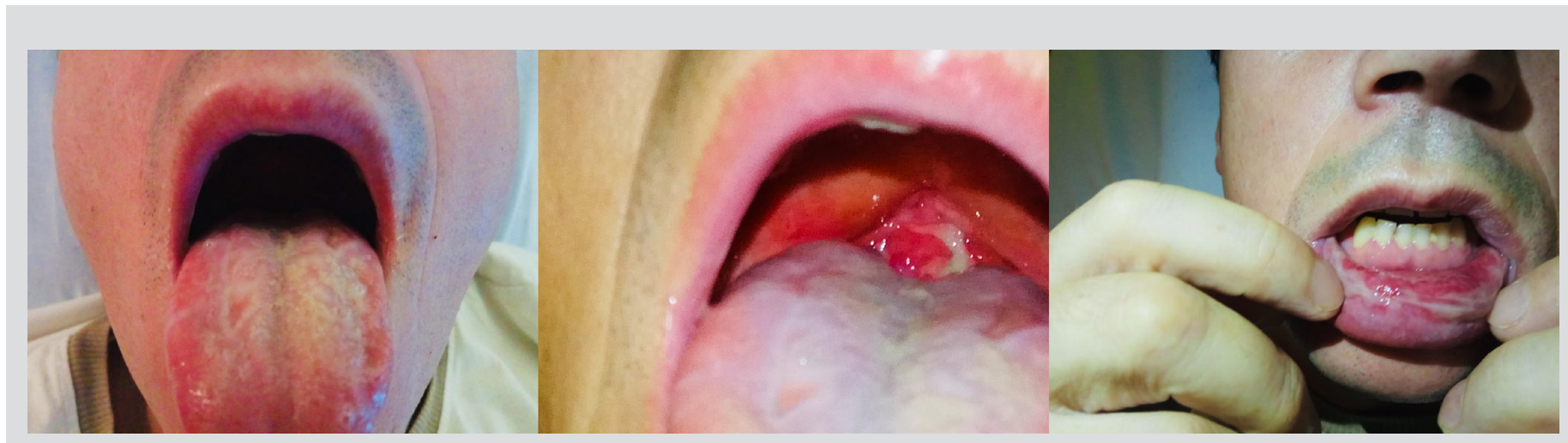

Figure 1. Oral mucositis with confluent ulcers on the oropharynx and hypopharynx

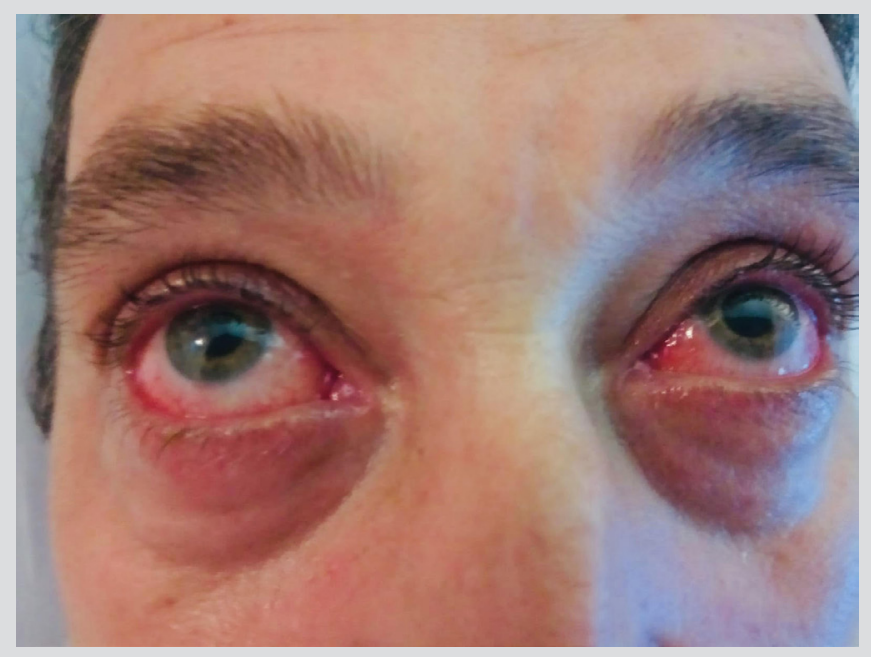

Figure 2. Conjunctival hyperaemia

During hospitalisation, the patient was treated with azithromycin $500 \mathrm{mg} / \mathrm{day}$ and gentamicin/dexamethasone eye drops (3/1 mg/ml) four times per day. Supportive care was given, including intravenous hydration and pain management with systemic analgesics. An oral suspension of nystatin and lidocaine was applied for oral mucosa care and artificial tear drops were applied for ocular lubrication. Due to the odynophagia, the patient received an individualised diet of semi-liquid food with protein-caloric supplementation. On the 7th day of hospitalisation due to persistence of the exuberant mucositis and conjunctivitis, systemic corticosteroid therapy with methylprednisolone 1 $\mathrm{mg} / \mathrm{kg} / \mathrm{day}$ was initiated. There was a favourable clinical evolution and the patient was discharged on the 13th day with mild mucosal lesions and mild conjunctival hyperaemia. One week after discharge the patient showed complete remission of the lesions. 


\section{DISCUSSION}

$\mathrm{MP}$ is a common cause of respiratory infections. In $25 \%$ of cases there are extrapulmonary complications, namely mucocutaneous lesions ${ }^{[1-5]}$. The MP spectrum of dermatological manifestations is varied and includes Raynaud's disease, erythema nodosum, Kawasaki disease, erythema multiforme (EM) and Stevens-Johnson syndrome/toxic epidermal necrolysis (SJS/TEN) ${ }^{[2-5]}$. Mucositis with or without scarce cutaneous involvement associated with MP infection is rare. Previously this clinical condition was classified within the epidermolytic dermopathy spectrum, being classified as 'atypical SSJ', 'incomplete SSJ' or 'Fuchs syndrome' [1,2,4]. A recent systematic review concluded that this condition is actually a distinct entity called Mycoplasma pneumoniae-induced rash and mucositis (MIRM) ${ }^{[1]}$. The MIRM characteristics include predominance of mucosa involvement (two or more mucosal sites) and scarce or absent cutaneous involvement (when present it manifests as a vesiculobullous or targetoid eruption), appearance at a younger age, a favourable prognosis with rare recurrence and a low mortality rate ${ }^{[1-3]}$. The pathophysiology of MIRM is unclear. It is thought to be caused by the production of immunoglobulins due to the proliferation of B cell clones which leads to the deposition of immune complexes in the mucocutaneous tissues. This pathophysiology is distinct from the pathophysiology of EM and SJS/TEM, as the latter are both mediated by a type IV delayed hypersensitivity reaction ${ }^{[1-3]}$. In the present case, the presence of a combination of factors enabled the diagnosis of MIRM: exclusive mucosal involvement, clinical and radiological findings of an atypical pneumonia and positive MP serology. This case also had a similar clinical course to other cases described in the literature in which respiratory symptoms preceded oral and ocular lesions ${ }^{[4,5]}$.

There are noguidelines for MIRM treatment but most of the cases described in the literature weretreated with antibiotics, immunomodulators (corticoid and/ or intravenous immunoglobulins) and supportive care ${ }^{[1-5]}$. Although antibiotic treatment directed at MP eliminates the causative agent and limits the duration and severity of the pulmonary disease, it is unclear whether the incidence or severity of the mucocutaneous eruption is reduced ${ }^{[1]}$. Macrolides are the most widely used antibiotics, mainly because of their immunomodulatory effect ${ }^{[5]}$. Although the role of immunomodulatory therapies is not clear, there is clinical evidence of the benefits of these drugs for the treatment of severe mucositis in reducing complications associated with mucous membrane adhesions ${ }^{[1]}$.

In the present case, the positive clinical evolution with total resolution of lesions is consistent with the diagnosis of MIRM. Recognition of MIRM as an independent entity has raised awareness and facilitated diagnosis and timely initiation of appropriate treatment.

\section{REFERENCES}

1. Canavan TN, Mathes EF, Frieden I, Shinkai K. Mycoplasma pneumoniae-induced rash and mucositis as a syndrome distinct from Stevens-Johnson syndrome and erythema multiforme: a systematic review. J Am Acad Dermatol 2015;72:239-245.

2. Santos RP, Silva M, Vieira AP, Brito C. Mycoplasma pneumoniae-induced rash and mucositis: a recently described entity. BMJ Case Rep 2017; doi: $10.1136 /$ bcr-2017-220768.

3. Martinez-Pérez M, Imbernón-Moya A, Lobato-Berezo A, Churruca-Grijelmo M. Mycoplasma pneumoniae-induced mucocutaneous rash: a new syndrome distinct from erythema multiforme? Report of a new case and review of the literature. Actas Dermosifiliogr 2016;107:e47-e51.

4. Vujic I, Shroff A, Grzelka M, Posch C, Monshi B, Sanlorenzo M, et al. Mycoplasma pneumoniae-associated mucositis-case report and systematic review of literature. J Eur Acad Dermatol Venereol 2015;29:595-598.

5. Meyer Sauteur PM, Goetschel P, Lautenschlager S. Mycoplasma pneumoniae and mucositis-part of Stevens Johnson syndrome spectrum. J Dtsch Dermatol Ges 2012;10:740746. 